\title{
Magnetic instrumentation and other applications of magnets in NOTES
}

\author{
Jarek Kobiela', Szymon Grymek², Magdalena Wojanowska', Marek Łubniewski², Wojciech Makarewicz', \\ Sebastian Dobrowolski', Andrzej J. Łachiński ${ }^{1}$, Zbigniew Śledziński ${ }^{1}$ \\ 1Department of General, Endocrine and Transplant Surgery, Medical University of Gdansk, Poland \\ 2Department of Machine Design and Exploitation, Faculty of Mechanical Engineering, Technical University of Gdansk, Poland
}

Videosurgery and Other Miniinvasive Techniques 2012; 7 (2): 67-73 DOI: 10.5114 /wiitm.2011.25665

\begin{abstract}
Modern surgery is developing towards a minimally invasive approach. To minimize the trauma the number of ports is either limited as in single incision laparoscopic procedures or ports are introduced through natural orifices as in natural orifice translumenal endoscopic surgery (NOTES). To provide surgeons with appropriate instrumentation novel technologies are employed involving magnets. This article summarizes the theoretical background, technology and currently developed magnetic instrumentation for NOTES, laparoscopic surgery and endoscopy. Moreover, current limitations and future goals are addressed to outline the prospects for use of magnetic instrumentation in the surgery of tomorrow.
\end{abstract}

Key words: natural orifice translumenal endoscopic surgery, magnetic instrumentation, surgical technology.

\section{Introduction}

Magnets have been used by humans since their discovery 10 centuries B.C. Their specific power and action have caused curiosity and fear at the same time. Over the centuries magnets have been employed in many fields of human life, among others by ancient medical doctors. Nowadays magnets and magnetic fields also have a wide range of practical medical applications. These applications include radiology (magnetic resonance imaging - MRI), physiotherapy (treatment of chronic pain), neurology and psychiatry (treatment of Parkinson's disease and depression with transcranial magnetic stimulation TMS). Recently attempts are being made to implement magnets in surgical instrumentation.

The development of laparoscopic surgery over the last two decades has revolutionized modern surgery. Laparoscopy is known for numerous advantages such as reduced postoperative pain, improved cosmesis and shortened recovery time. The surgeon inserts multiple ports to introduce instruments for appropriate visualization and manipulation. Increasing the number of ports contributes to postoperative pain and diminished cosmesis, and carries a risk of bleeding, hernia formation, etc. These effects can limit the advantage of laparoscopy, and this is why currently, to further minimize surgical injury, novel techniques are available including the most innovative NOTES (natural orifice translumenal endoscopic surgery) and SILS (single incision laparoscopic procedures) [1, 2]. The greatest challenge for this innovative field is the development of novel instrumentation that will overcome the shortcomings of conventional instruments [3]. Classic flexible endoscopic platforms which are currently 
available do not provide suitable stability, force and torque, provide inadequate manoeuvrability and lack triangulation [4, 5]. These problems have led medical engineers to search for new technical solutions which will enable further development of these new minimally invasive techniques. One of the new technical developments was the TransPort EndoSurgical Operating Platform (USGI Medical, San Clemente, CA, USA), which is a four-channel platform flexible scope based on the Shapelock locking technology [6]. The TransPort contains four operating channels that accommodate two $6-\mathrm{mm}$ and two 4-mm instruments. It is flexible, allowing for insertion of the device through the gastrointestinal tract. After the positioning, the base of the endoscope can be locked into position while allowing the distal operating tip to be steered freely. This Operating System is available commercially [2]. It seems however that the perspective for further improvement of flexible endoscopes is limited. This is why novel technologies including the Magnetic Anchoring and Guidance System (MAGS) are extensively studied to provide maximal ergonomy and efficiency with minimal injury.

\section{Magnets and magnetic field - the theory and nomenclature}

A magnet is a material or object that produces a magnetic field. A magnetic field (usually denoted B) is defined as a vector field which surrounds magnets and electric currents. The field has its own energy and can be detected by virtue of the fact that it exerts a force on moving electric charges and on magnetic materials. Such materials are known as ferromagnetic (e.g. iron, nickel, cobalt, some rare earth metals, some alloys).

A magnet can be:

1) permanent - made from a material that stays magnetized (ferromagnetic);

2) an electromagnet - made from a coil of an electric conductor which acts as a magnet when (and only when) an electric current passes through it.

Gauss's law for magnetism (one of Maxwell's equations) states that the magnetic field $B$ has divergence equal to zero. This means that magnetic monopoles do not exist and the basic entity for magnetism is the magnetic dipole. A magnet has two different ends called north and south magnetic poles, which cannot be separated. A magnet produces a magnetic field and it responds to magnetic fields. When it is put into a magnetic field produced by a different source it is subject to a torque tending to orient the magnetic moment parallel to the field and a force driving it in one direction or another, according to the positions and orientations of the magnet and source.

Generally, calculating the attractive or repulsive force between two magnets is a complex procedure. The force value depends on the shape, magnetization, orientation and separation of the magnets.

Theoretically, the force between two magnetic poles is given by:

$$
F=\frac{\mu q_{m 1} q_{m 2}}{4 \times \pi \times r^{2}}
$$

where $F$ is force; $q_{m 1}$ and $q_{m 2}$ are the magnitudes of magnetic poles, $\mu$ is the permeability of the intervening medium; $r$ is the separation.

A pole distribution for real magnets makes the above formula useless. In some cases more useful formulae can be presented:

1) two nearby attracting surfaces of area $A$ and equal but opposite magnetizations $M$ :

$$
F=\frac{\mu_{0}}{2} \times A \times M^{2}
$$

where $A$ is the area of each surface, $M$ is their magnetization, $\mu_{0}$ is the permeability of space;

2) two identical cylindrical bar magnets placed end to end:

$\mathrm{F}=\left[\frac{B_{0}^{2} \times A^{2} \times\left(L^{2}+R^{2}\right)}{\pi \times \mu_{0} \times L^{2}}\right] \times\left[\frac{1}{x^{2}}+\frac{1}{(x+2 \times L)^{2}}-\frac{2}{(x+L)^{2}}\right]$

where $B_{0}$ is the magnetic flux density very close to each pole, $A$ is the area of each pole, $L$ is the length of each magnet, $R$ is the radius of each magnet, $x$ is the separation between the two magnets.

In practical application the force must be measured during an experiment. Permanent magnets having technical applications are listed in Table I. Some vendors show the magnets (points 3 to 5) even stronger - see Figure 1. A permanent magnet must be made as a monolith. This means that 2 linked $70 \times 20$ magnets do not generate a field as strong as a single $70 \times 40$ magnet (magnets are not batteries). 
Table I. Types of permanent magnets having technical applications

\begin{tabular}{|lc|}
\hline Permanent magnet types & Magnetic energy product \\
\hline Ferrite magnet (BaFe12O19 or SrFe12O19) & Up to 3 times higher than ferrite magnet \\
\hline Alnico magnet (Al-Ni-Co-Fe) & Up to 5 times higher than ferrite magnet \\
\hline Samarium-cobalt magnet (Sm-Co) & Up to 4 times higher than ferrite magnet \\
\hline Neodymium magnet (NIB magnet) plastic-bonded (Nd-Fe-B) & $\begin{array}{c}\text { Up to } 20 \text { times higher than ferrite magnet } \\
\text { (the strongest available) }\end{array}$ \\
\hline Neodymium magnet (NIB magnet) sintered (Nd-Fe-B) & \\
\hline
\end{tabular}
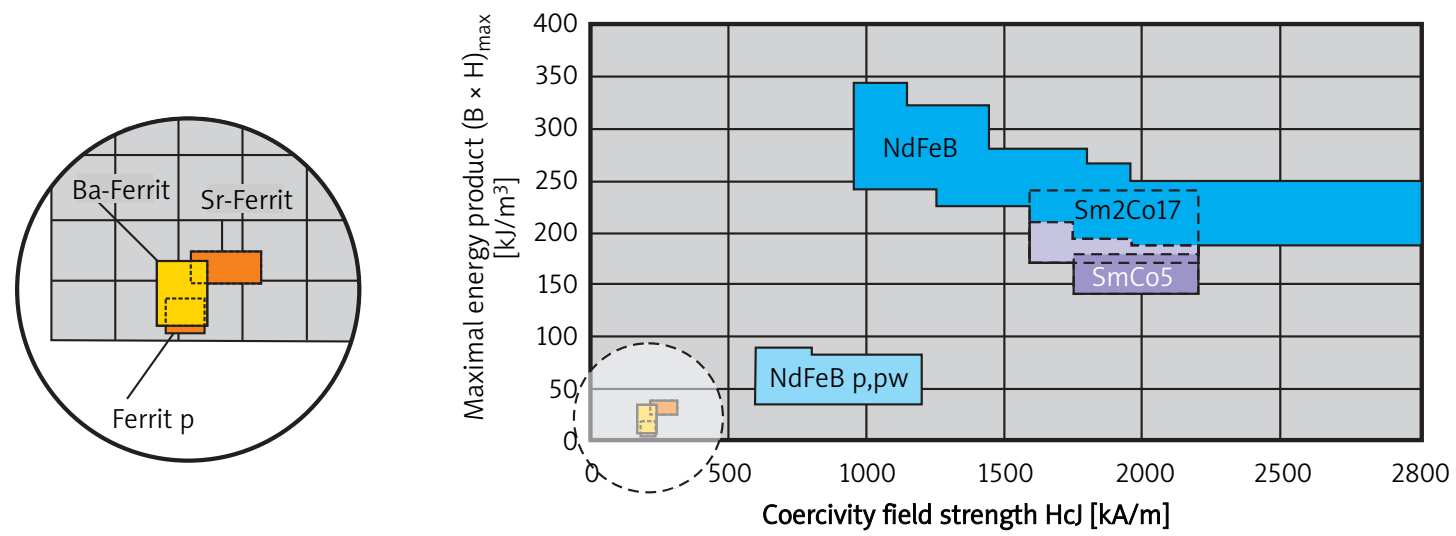

$p$-plastic-bonded injected, $p w$ - plastic-bonded pressed

Figure 1. Coercivity field strength and maximal magnetic energy for commercially available permanent magnets. Adopted with permission from www.neodym.pl

Theoretically, the force generated by a single solenoid with a core is given by:

$$
F=\frac{12 \times n^{2} \times k \times \mu_{0} \times A}{2 \times g^{2}}
$$

where $I$ is the electric current in a solenoid, $n$ is a number of coil turns, $k$ is relative permeability of the solenoid core, $\mu_{0}$ is the permeability of a vacuum, $A$ is the cross-sectional area of the coil's core, $g$ is the gap between core and armature.

For the problem of magnetic instrument anchoring a permanent magnet should be used rather than an electromagnet. The main disadvantages of an electromagnet for this application are:

1) it works (produces force) when an electric current passes through it (no current - no force - no anchoring);

2) every current pass generates thermal energy;
3) DC current must be applied to avoid force changes in time, varying the magnetic field.

\section{Magnetic Anchoring and Guidance System Technology}

Magnetic Anchoring and Guidance System (MAGS) is a new technology which deploys multiple instruments through a single port which can be located transumbilically, transvaginally or in any other area [7]. Initially it was developed mainly to minimize the number of trocars in laparoscopic procedures but quickly attempts were made to use this method in NOTES and endoscopic procedures.

Magnetic Anchoring and Guidance System instruments are positioned in the peritoneal cavity and controlled by externally placed magnets. The MAGS working prototype consists of a stack of neodymiumiron and boron (Nd-Fe-B) permanent magnets. They 
are focused and shielded to optimize strength and generate sufficient coupling forces to control $25-45 \mathrm{~g}$ instruments $[7,8]$. This strength was determined across various distances of air gaps, ex vivo porcine abdominal walls and commercially purchased bovine muscle of various thicknesses [7, 9]. Experimental studies have proven that abdominal wall thickness in excess of $1.5 \mathrm{~cm}$ limits the effectiveness of the magnetic anchoring [10] so stronger magnets (Figure 1) may be needed to maintain adequate instrument control in humans with a thicker abdominal wall. Currently this technology would be adequate for thin and paediatric patients.

Over several years new prototype MAGS instruments have been designed, manufactured and evaluated in vivo while performing procedures on experimental animals. The experience grows and the instrumentation is becoming more sophisticated and advanced.

Initially two types of passive tissue retractors were designed which can be introduced through a single, classic $12-\mathrm{mm}$ trocar and then anchored by externally placed magnets. The first type is a sling retractor consisting of latex tubing which is used for elevating and moving organs such as the liver or spleen. It is attached to two internal MAGS platforms and by manipulating the pair of external anchors, the sling could be positioned under the organ to elevate it. The latter one is a paddle-type retractor which provides lateral retraction $[7,9]$.

The next step was to design the pneumatically powered robotic arm which carries a conventional hook cautery. The arm was designed to mimic the motions of a cautery hook operated by the surgeon's right hand. It has three joints: the first raises and lowers the arm; the second joint acts like an elbow and moves only to the left; and the third allows for telescoping the cautery. To control the robotic arm an offthe-shelf gaming joystick is used as an input device. After positioning and anchoring to the abdominal wall by an externally placed magnet, the joystick is used to activate the robotic arm to accomplish tissue incision $[7,10]$. There is also a more advanced robotic platform for NOTES procedures which consists of a miniature in vivo robot inserted fully into the peritoneal cavity through a natural orifice, a surgeon control console and an external magnetic handle. The body of the robot contains embedded magnets that couple with magnets in an external handle or the surgeon console for attachment of the robot to the interior abdominal wall. There are two arms divided into two smaller upper and lower ones. The mass of the robot is approximately $110 \mathrm{~g}$. The surgeon console consists of two analogue joysticks for controlling the manipulation of the robot arms. One joystick also has two pushbutton controls for opening and closing the grasper jaws. Between the two joysticks a colour LCD monitor is located to display the video from the robot cameras. A foot pedal is used to activate the cautery [4]. In addition a MAGS camera was created by putting a commercially available miniature camera into a customized aluminium enclosure attached to a pair of internal MAGS platforms. It weighs $35 \mathrm{~g}$ [10]. Manipulation of the pair of external magnets causes the horizontal orientation of the camera. By pushing the external magnets apart or together it is possible to actively point the camera lens downward or upward.

\section{Applications}

So far MAGS platforms have been used to perform and facilitate several minimally invasive surgical procedures including NOTES, single incision laparoscopic surgery and interventional endoscopic procedures.

Initially, the MAGS instrumentation was used to perform porcine nephrectomies with only two trocars: a $15 \mathrm{~mm}$ 'light' trocar and a $5 \mathrm{~mm}$ conventional trocar [7, 9]. Gradually single trocar laparoscopic left nephrectomies were successfully performed on a porcine model. Magnetic Anchoring and Guidance System instrumentation was deployed into the abdomen through a single $12-\mathrm{mm}$ diameter, transumbilical trocar, then the 'light' trocar was inserted through the same incision along the wires controlling the MAGS camera and the robotic cautery. During these procedures a conventional laparoscopic grasper and stapler were used and introduced through the 'light' trocar. No intraoperative complications were encountered while performing these procedures and blood loss was minimal $[6,10]$. Further attempts have been made to utilize the MAGS technology in NOTES procedures to provide ergonomy and address the shortcomings of the classic flexible endoscopic instrumentation. The MAGS instrumentation can be assisted by the magnetic retraction system for NOTES procedures.

The magnetic retraction system consists of an external magnetic assembly and internal magnets attached by suture to endoscopic clips. It is reported 
that this system was used in two procedures on porcine models: transcolonic endoscopic cholecystectomy and manipulation and stabilization of mesh for transcolonic endoscopic ventral hernia repair [11]. This magnetic retraction system was able to lift the hepatic lobe and provide adequate exposure of the gallbladder, better visualization and identification of the cystic duct and artery and facilitate dissection. In transcolonic endoscopic ventral hernia repair the magnetic retraction system facilitates the positioning and stabilization of the mesh compared to procedures with non-magnetic-assisted implantation [11].

Completely transvaginal NOTES cholecystectomies were performed successfully on porcine models. After creating the vaginotomy, a rigid access port was inserted into the peritoneal cavity and $\mathrm{CO}_{2}$ pneumoperitoneum was maintained. Magnetic Anchoring and Guidance System instruments were deployed through the port and then held in place by external handheld magnets. During these procedures a tissue retractor (flexible graspers) and a cautery dissector were used. The classic gastroscope was used for visualization [8]. Magnetic Anchoring and Guidance System technology was also used for transgastric cholecystectomies using a miniature robot which is described above. The robot was introduced to the peritoneal cavity through transgastric insertion and attached to the interior abdominal wall using the interaction of magnets in the robot body with magnets in the external magnetic handle. During the procedure the robot was repositioned to optimize the tissue retraction and visualization [4].

\section{Magnetic-Anchor-Guided Endoscopic Submucosal Dissection}

Magnets have also been employed in endoscopic procedures. It has been reported that the magnetic anchor was used successfully in the novel technology called MAG-ESD (magnetic-anchor-guided endoscopic submucosal dissection) for endoscopic treatment of early gastric cancer in humans [12]. The magnetic anchor consists of 3 parts: a hand-made magnetic weight, made of magnetic stainless steel, microforceps and a connecting thread. It is controlled by external electromagnets. It has been reported that MAG-ESD is a feasible and safe method that allows excellent visualization by suitable tissue tension and facilitates standard ESD [12].

\section{Magnetic anastomoses}

Neodymium magnets have been safely used in endoscopic gastroenteric anastomosis [11]. Magnetcontrolled approaches were concluded to be promising in biliary and vascular anastomoses (although the latter involves permanent implantation) [13-16]. In contrast, magnetic hindgut anastomosis devices have been limited by leakage and problems with device expulsion [17]. Magnetic gastrojejunostomy has been reported, but these anastomoses demonstrated limited primary patency and required endoluminal stents to preserve function $[18,19]$. Attempts are being made to use temporary magnets in performing sutureless compression anastomoses [13] called magnamosis. So far they have been successfully used in porcine models [13].

\section{Current limitations and future goals}

Magnetic Anchoring and Guidance System is a novel technology which can greatly contribute to the development of minimally invasive surgery. However, several current limitations must be addressed before application to human procedures. First some improvements must be made to current instrumentation.

It was noted that the MAGS camera although experimental was designed for non-medical use and it was inferior to the classic laparoscopic camera [7]. It has a fixed focus which makes obtaining a sharp image more difficult than in laparoscopic procedures. A future goal is to design MAGS cameras with parameters similar to those which are currently used in laparoscopy, which means High Definition. The next goal is to provide a wireless power source and signal transmission.

Other instruments also need some modernization and improvement in parameters. The joystick-controlled motions of the robotic cautery are not smooth enough and difficulty has been encountered with telescoping the arm. However, the robotic arm had sufficient force to elevate and incise tissues and the hook cautery transmitted the current flawlessly [7-10].

Another limitation which must be resolved before the implementation of MAGS in human procedures is reinforcement of the anchoring power of the magnets, because outgoing parameters are not compatible with the thickness of adults' abdominal wall. It was reported that during some procedures on porcine models surgeons had difficulties with the 
coupling strength of the MAGS instruments (magnetic miniature robots) using currently available permanent magnet configurations even though the thickness of the porcine models was less than $2.5 \mathrm{~cm} \mathrm{[4]}$ (see formula 3). For this reason the NOTES intervention had to be converted to laparoscopy.

Additionally a future goal is to provide a local power source and wireless controls to enable instruments to be operated independently from their deployment site. Nowadays, all tools are supplied from an external power source and controlled with wired technology. Taking into consideration the time of a therapeutic procedure and power requirement from surgical tools there seems to be no commercially available wireless power source feasible for the application at the moment. The voltage and capacity of modern batteries still seem too low to supply all tools (especially the electric knife). Wireless control is not a serious problem because although in static magnetic fields the radio control signal is weakened, practically it remains undisturbed. Classic mechanical control has a dynamic loop (feedback). The operator "feels" the force applied. Both the electric control and the wireless control have no such feedback. Only the displacement of the tool is controlled. Force feedback can be artificially added by electronic force sensing and servo simulated feedback applied to controls, but this adds a lot to the overall complexity of tools and can diminish the overall reliability.
Another problem which has to be taken into consideration is the impact of magnets and magnetic fields on human tissues. It is assumed that in anchoring applications a static magnetic field is applied. Human tissues have a very low level of susceptibility to a static magnetic field and therefore the health hazard associated with exposure to this field is low. However, in some limited cases the magnetic field can pose a serious risk. Important risks and concerns for use of magnetic fields in humans are summarized in Table II.

The next problem which has to be taken into consideration is the interaction between magnets and other instrumentation used during procedures. To minimise or avoid interactions between working mechanical parts and magnetic fields it is necessary to design and manufacture them from non-ferrous materials (paramagnetic or diamagnetic). Additionally, they must not interact with human tissue. Considering the above, the most obvious materials of choice for this application are:

- titanium alloys,

- certain grades of stainless steel,

- plastics,

- copper-based alloys (with additional coat),

- certain grades of technical ceramics.

The general concept of the tools is being developed in two approaches: one is a set of separate, independently anchored and controlled tools; anoth-

Table II. Important risks and concerns for use of magnetic fields in humans

A ferromagnetic foreign body in human tissue can interact with the magnetic field (additional forces, magnetisation of the body, change of the magnetic field).

Human tissues and body fluids are conductive to electricity; thus electro-dynamic interactions with moving electrolytes (especially blood flow) induce electric fields and currents in a tissue or medium. Passage of electric current through live tissues can cause adverse effects (e.g. blood clotting), so the magnets should be as far as possible from main blood vessels, in which quick passage of blood in large quantities occurs.

All electrically powered equipment should be protected from the magnetic field.

A magnetic field can change kinetics of chemical reactions in body cells. The influence is not well recognised but seems to be less important.

Electrical control signals, as well as radio wireless signals, can be disturbed by magnetic fields.

Because of their chemical properties all permanent magnets (especially neodymium type) should be isolated from contact with tissues and/or fluids inside the body. The shielding material must be inert to the human body and non-obstructive to the magnetic field, e.g. gold or epoxy resin.

All interacting magnets should have a close contact over a large surface area. These two combined should help reduce the contact stress and possible risk of harm. 
er is an "all in one" solution - anchored together but controlled independently. We suggested an approach where separate tools are supported on a common, magnetically anchored working platform. The tools are controlled independently and can be moved over the platform. Such an approach gives greater working space, allows more accurate control and minimises the influence of low rigidity and heterogeneity of human tissues.

\section{Summary}

Modern surgery is developing towards a minimally invasive approach. To minimize the trauma, the number of ports is either limited as in SILS or ports are introduced through natural orifices as in NOTES. To provide surgeons with appropriate instrumentation, novel technologies are employed involving magnets. The clinical application of magnets has been proved in several surgical settings and will probably further develop with progress of NOTES, laparoscopic surgery and endoscopy.

\section{References}

1. Michalik M, Frask A, Orłowski M. NOTES (Natural Orifice Translu minal Endoscopic Surgery) - operacje przez naturalne otwory ciała. Videosurgery and Other Miniinvasive Techniques 2007; 2: 98-102.

2. Kurpiewski W, Pesta W, Kowalczyk M, et al. SILS cholecystectomy - our first experiences. Videosurgery and Other Miniinvasive Techniques 2009; 4: 91-4.

3. Witzling $M$, Michalik M, Pawlak M. NOTES in patients treated in intensive care units - the new challenge Videosurgery and Other Miniinvasive Techniques 2009; 4: 154-7.

4. Lehman AC, Dumpert AJ, Nathan AA, et al. Natural orifice cholecystectomy using a miniature robot. Surg Endosc 2009; 23 260-6.

5. Hać S, Kobiela J, Proczko-Markuszewska M, et al. Cholecystektomia z dostępu przez pochwę - NOTES - pierwsze starcie. Videosurgery and Other Miniinvasive Techniques 2008; 3: 139-41.

6. Swanstrom LL, Whiteford M, Khajanchee Y. Developing essential tools to enable transgastric surgery. Surg Endosc 2008; 22: 600-4.

7. Zeltser IS, Cadeddu JA. Novel magnetic anchoring and guidance system to facilitate single trocar laparoscopic nephrectomy. Curr Urol Rep 2008; 9: 62-4.

8. Scott DJ, Shou-jiang Tang A, Raul Fernandez A, et al. Completely transvaginal NOTES cholecystectomy using magnetically anchored instruments. Surg Endosc 2007; 21: 2308-16.

9. Park S, Park S, Bergs RA, et al. Trocar-less instrumentation for laparoscopy: magnetic positioning of intra-abdominal camera and retractor. Ann Surg 2007; 245: 379-84.

10. Zeltser IS, Bergs R, Fernandez R, et al. Single trocar laparoscopic nephrectomy using magnetic anchoring and guidance system in the porcine model. J Urol 2007; 178: 288-91.
11. Ryou M, Thompson CC. Magnetic retraction in natural-orifice transluminal endoscopic surgery (NOTES): addressing the problem of traction and countertraction. Endoscopy 2009; 41: 143-8.

12. Gotoda T, Oda I, Tamakawa K, et al. Prospective clinical trial of magnetic-anchor-guided endoscopic submucosal dissection for large early gastric cancer (with videos). Gastrointest Endosc 2009; 69: 10-5.

13. Jamshidi R, Stephenson JT, Clay JG, et al. Magnamosis: magnetic compression anastomosis with comparison to suture and staple techniques. J Pediatr Surg 2009; 44: 222-8.

14. Muraoka N, Uematsu H, Yamanouchi E, et al. Yamanouchi magnetic compression anastomosis for bilioenteric anastomotic stricture after living-donor liver transplantation. J Vasc Interv Radiol 2005; 16: 1263-7.

15. Mimuro A, Tsuchida A, Yamanouchi E, et al. A novel technique of magnetic compression anastomosis for severe biliary stenosis. Gastrointest Endosc 2003; 58: 283-7.

16. Erdmann D, Sweis R, Heitmann C, et al. Side-to-side sutureless vascular anastomosis with magnets. J Vasc Surg 2004; 40: 505-11.

17. Jansen A, Brummelkamp WH, Davies GA, et al. Clinical applications of magnetic rings in colorectal anastomosis. Surg Gynecol Obstet 1981; 153: 537-45.

18. Cope C. Creation of compression gastroenterostomy by means of the oral, percutaneous, or surgical introduction of magnets: feasibility study in swine. J Vasc Interv Radiol 1995; 6: 539-45.

19. Cope C, Clark TW, Ginsberg G, et al. Stent placement of gastroenteric anastomoses formed by magnetic compression. J Vasc Interv Radiol 1999; 10: 1379-86. 